Cite this: J. Mater. Chem. A, 2018, 6, 19190

Received 31st May 2018

Accepted 10th September 2018

DOI: $10.1039 / c 8 t a 05132 a$

rsc.li/materials-a

\section{The finale of a trilogy: comparing terpolymers and ternary blends with structurally similar backbones for use in organic bulk heterojunction solar cells $\uparrow$}

\author{
Mary Allison Kelly, (D) a Qianqian Zhang, ${ }^{a}$ Zhengxing Peng, ${ }^{b}$ Victoria Noman, (D) \\ Chenhui Zhu, ${ }^{\mathrm{c}}$ Harald Ade ${ }^{\mathrm{b}}$ and Wei You (D) *a
}

Building on our previous works that compared the efficacy of terpolymers vs. ternary blends in improving the performance of bulk heterojunction organic solar cells, the final piece of this series of studies focuses on comparing terpolymer and ternary blends constructed with two polymers with structurally similar backbones (monoCNTAZ and FTAZ) yet markedly different open circuit voltage $\left(V_{\text {oc }}\right)$ values. Terpolymers and ternary blends of five different ratios were studied and the results demonstrate that while the overall performance of both the systems is similar, the ternary blends exhibit higher short circuit current $\left(J_{\mathrm{sc}}\right)$ values, while the terpolymers exhibit higher $V_{\text {oc }}$ values. Investigation of the charge transfer state using low-energy external quantum efficiency (EQE) indicates that the ternary blends are governed by a parallel-like mechanism, while the terpolymer does not follow this mechanism. The key morphological difference between the systems, as elucidated by resonance soft X-ray scattering (RSoXS), is the slightly smaller size $(\sim 60 \mathrm{~nm})$ of domains in the ternary blends compared to that of the terpolymer $(\sim 80 \mathrm{~nm})$, which may affect exciton harvesting in the terpolymer system and lead to lower $J_{\mathrm{sc}}$ values. In addition, a lower driving force for the formation of charge transfer (CT) state is also likely to contribute to the lower $J_{\mathrm{sc}}$ values in the terpolymer system. All together, the data show that structurally similar (perhaps even miscible) polymers still exhibit key differences in performance when paired in terpolymers vs. ternary blends and allow us to further illuminate the underlying mechanisms of such complex systems.

\section{Introduction}

While organic solar cells (OSCs) offer exciting developments in photovoltaic technology with their versatility and inexpensive fabrication, they continue to be hampered by the inherently narrow absorption window of the conjugated polymers. ${ }^{1}$ Two different strategies have thus far been used to incorporate multiple conjugated polymers (ideally with complementary absorption) into the bulk heterojunction (BHJ) layer to extend the absorption range of OSCs. One strategy is the ternary blend where two donor polymers and one acceptor molecule are physically mixed, and the other one is the terpolymer where three (or more) different structural units from two parent conjugated polymers are copolymerized together (i.e., chemically mixed). Both strategies have proven to be effective in

${ }^{a}$ Department of Chemistry, University of North Carolina at Chapel Hill, Chapel Hill, North Carolina, USA. E-mail: wyou@unc.edu

${ }^{b}$ Department of Physics and ORaCEL, North Carolina State University, Raleigh, North Carolina, USA

'Advanced Light Source, Lawrence Berkeley National Laboratory, Berkeley, California 94720, USA

$\dagger$ Electronic supplementary information (ESI) available: Synthetic details, GPC curves, elemental analysis, CV scans and CT state fittings. See DOI: 10.1039/c8ta05132a extending the absorbance of an organic solar cell and improving the performance. ${ }^{1-9}$ Recent efforts have yielded some of the highest efficiencies for organic solar cells (OSCs) utilizing these strategies. $^{3,10}$ In fact, one of the highest reported efficiency (14.1\%) of single junction OSCs was achieved with a ternary blend that included a polymer (PTB7-Th), a fullerene acceptor $\left(\mathrm{PC}_{71} \mathrm{BM}\right)$ and a non-fullerene acceptor $\left(\mathrm{CO}_{i} 8 \mathrm{DFIC}\right) .{ }^{11}$ However, the success of ternary blends as well as terpolymer-based solar cells is still largely an empirical process. Several recent studies have highlighted the importance of polymer compatibility and miscibility for working ternary blend OSCs; $;^{12-14}$ but given the complexity of these systems, further investigation is required.

In our previous studies, we have introduced two systems where we directly compared a terpolymer with a ternary blend of its parent polymers; ${ }^{15,16}$ such practices have allowed us to understand the fundamental mechanisms in both the systems. Initially, we investigated the terpolymer of two parent polymers PBnDT-DTBT (abbreviated as DTBT) and PBnDT-HTAZ (i.e., HTAZ), which shared the same donor moiety BnDT. These two polymers, when used in a ternary blend, showed improved performance over their binary blends. ${ }^{15,17,18}$ In this first system, there was a marked difference in the performance of OSCs made of the terpolymer and the ternary blend. The terpolymer showed improved open circuit voltage $\left(V_{\text {oc }}\right)$ but lower short 
circuit current $\left(J_{\mathrm{sc}}\right)$ compared to those of the ternary blend, possibly because the difference in the acceptor moiety (i.e., DTBT unit vs. HTAZ unit) could lead to immiscibility of these two parent polymers (DTBT and HTAZ). These observations suggested that there can be significant differences between a terpolymer and ternary blend.

In the second study, we focused on a system with similar backbones by comparing PBnDT-HTAZ (i.e., HTAZ) and PBnDTFTAZ (i.e., FTAZ). ${ }^{16}$ We had previously demonstrated that random copolymers of these two parent polymers (which differ solely by the addition of two fluorine atoms) displayed photovoltaic properties between those of the parent polymers. ${ }^{19}$ In this follow-up study, we directly compared the $1: 1$ physical blend of HTAZ and FTAZ with the terpolymer (also $1: 1$ feed ratio) and a newly synthesized monofluorinated copolymer PBnDT-monoFTAZ (monoF). Surprisingly, in this system, there was no observable difference in the device characteristics between the terpolymer ( $1: 1$ feed ratio) and the ternary blend (1:1 physical blend). This is a direct contrast to our previous study and serves to highlight the diversity of photovoltaic behavior in ternary blends. More importantly, this second study suggested a plausible guideline: if the polymers are similar/ miscible, they are more likely to have similar behavior in the ternary blend and the terpolymer. Evidently, there have already been several examples demonstrating that the compatibility of the polymers is key to a successful ternary blend. ${ }^{6,12,14,20,21}$

Building on these studies, we have moved on to what may prove to be an ideal terpolymer/ternary blend system by selecting PBnDT-monoCNTAZ and PBnDT-FTAZ (abbreviated as monoCNTAZ and FTAZ in Fig. 1) as our parent polymers. Unlike DTBT and HTAZ, these two polymers share a structurally similar acceptor moiety (benzotriazole, TAZ) in addition to the same donor moiety BnDT. Thus, these two polymers would likely be 'miscible', from a structural point of view. However, the switch from a fluorine substituent to the stronger electron withdrawing cyano group results in a smaller band gap for monoCNTAZ (1.85 eV). ${ }^{22}$ Thus, this system is an improvement on the HTAZ:FTAZ system, where the two polymers show almost identical absorption. The current system (monoCNTAZ and FTAZ) has the potential for increased $J_{\mathrm{sc}}$ from extended absorbance, a key motivator behind both ternary blends and terpolymers. ${ }^{7,23}$ Furthermore, the highest occupied molecular orbital (HOMO) level of monoCNTAZ is slightly deeper than that of FTAZ $(-5.58 \mathrm{eV}$ vs. $-5.45 \mathrm{eV})$, which results in a higher $V_{\mathrm{oc}}$ for
monoCNTAZ-based devices than that of the FTAZ-based ones (0.935 V vs. $0.811 \mathrm{~V}$, vide infra, Table 1$)$.

With the interesting features of this new system, we designed our study to compare the terpolymer of these two parent polymers with ternary blends of five monoCNTAZ:FTAZ (mC:F) ratios $(9: 1,7: 3,1: 1,3: 7$ and $1: 9)$ to further explore the differences between terpolymer and ternary blend. Should the polymers show compatible and cooperative behavior, there may be a corresponding overall increase in the photovoltaic performance of the ternary blends, as has been demonstrated in the literature. ${ }^{24-27}$ Furthermore, the significant difference in $V_{\text {oc }}$ between the bulk heterojunction (BHJ) solar cells based on these parent polymers provides an ideal pairing to study the effect of terpolymer $v s$. ternary blend on $V_{\mathrm{oc}}$, which is an often debated subject in ternary blend OSCs especially since the terpolymer mimics the close intermixing required for the suggested alloy model. ${ }^{12,28}$

Not surprisingly, upon physically blending monoCNTAZ and FTAZ (i.e., the ternary blend), we found that the $V_{\text {oc }}$ of the ternary blend OSCs is composition-dependent, which is the hallmark of a working parallel-like or alloy model bulk heterojunction. ${ }^{2}$ However, the overall performance is the average of both the parent polymers and there is no enhancement in efficiency (which is only possible if the polymers are compatible and behave cooperatively). On directly comparing the performance of the terpolymer and the ternary blend OSCs at each monoCNTAZ:FTAZ (mC:F) ratio, the overall performance was interestingly very similar but with marked differences in the $V_{\text {oc }}$ and $J_{\text {sc }}$. Moreover, the fill factor (FF) was maintained for both the terpolymer and the ternary blend OSCs. With a more significant change to the backbone (i.e., fluorine to cyano) than in our previous study (i.e., hydrogen to fluorine in the case of HTAZ and FTAZ), there is a marked difference between the terpolymer and the ternary blend despite the compatibility of these polymers. These results highlight the complexity of such systems, and also demonstrate that high performance ternary blends are within our reach as we unravel more of the underlying operating principles.

\section{Experimental methods}

All chemicals were purchased from commercial source (SigmaAldrich, Fisher, Matrix, etc.) and used as received unless specified otherwise. Tris(dibenzylideneacetone)-dipalladium(0)-
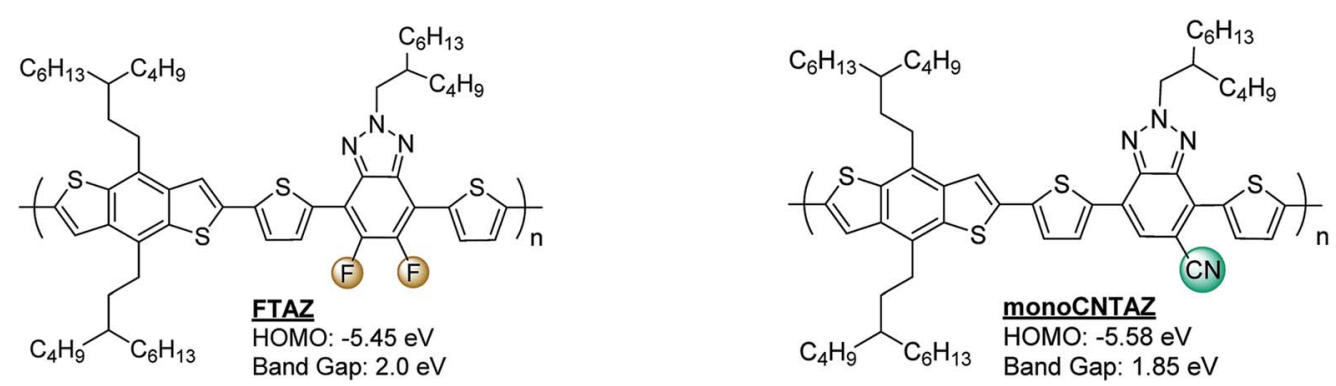

Fig. 1 The structures, HOMO energy levels, and bandgaps for the two parent polymers used in this study. 
Table 1 The photovoltaic performance of all the systems investigated in this study

\begin{tabular}{|c|c|c|c|c|c|}
\hline Polymer & Thickness (nm) & $J_{\mathrm{sc}}\left(\mathrm{mA} \mathrm{cm} \mathrm{cm}^{-2}\right)$ & $V_{\mathrm{oc}}(\mathrm{V})$ & $\mathrm{FF}(\%)$ & PCE (\%) \\
\hline monoCNTAZ & $202 \pm 1$ & $13.30 \pm 0.43$ & $0.935 \pm 0.001$ & $68.9 \pm 2.0$ & $8.57 \pm 0.34$ \\
\hline mC:F $9: 1$ & $202 \pm 1$ & $13.44 \pm 0.2$ & $0.920 \pm 0.002$ & $66.9 \pm 1.4$ & $8.25 \pm 0.21$ \\
\hline mC:F $1: 1$ & $202 \pm 15$ & $12.95 \pm 0.14$ & $0.867 \pm 0.003$ & $68.2 \pm 2.4$ & $7.66 \pm 0.33$ \\
\hline mC:F $3: 7$ & $218 \pm 13$ & $12.71 \pm 0.37$ & $0.850 \pm 0.003$ & $68.7 \pm 1.1$ & $7.42 \pm 0.21$ \\
\hline mC:F $1: 9$ & $202 \pm 46$ & $11.80 \pm 0.12$ & $0.828 \pm 0.002$ & $72.1 \pm 1.3$ & $7.05 \pm 0.13$ \\
\hline co-mC-F $7: 3$ & $209 \pm 8$ & $12.36 \pm 0.43$ & $0.904 \pm 0.003$ & $69.5 \pm 2.0$ & $7.77 \pm 0.31$ \\
\hline co-mC-F $1: 1$ & $227 \pm 5$ & $12.37 \pm 0.32$ & $0.899 \pm 0.001$ & $66.7 \pm 2.8$ & $7.42 \pm 0.37$ \\
\hline co-mC-F $3: 7$ & $240 \pm 11$ & $11.98 \pm 0.31$ & $0.863 \pm 0.002$ & $68.7 \pm 3.9$ & $7.11 \pm 0.5$ \\
\hline co-mC-F $1: 9$ & $244 \pm 20$ & $11.57 \pm 0.38$ & $0.834 \pm 0.001$ & $68.5 \pm 3.0$ & $6.61 \pm 0.37$ \\
\hline
\end{tabular}

chloroform adduct $\left(\mathrm{Pd}_{2} \mathrm{dba}_{3} \cdot \mathrm{CHCl}_{3}\right)$ was purchased from Sigma-Aldrich and was recrystallized in chloroform/acetone. Tri $(o$-tolyl $)$ phosphine $\left(\mathrm{P}(o \text {-tol })_{3}\right)$ was recrystallized in hexanes.

Microwave assisted polymerizations were conducted in a CEM Discover Benchmate microwave reactor. Gel permeation chromatography (GPC) measurements were performed at $150{ }^{\circ} \mathrm{C}$ on a Polymer Laboratories PL-GPC 220 instrument using 1,2,4-trichlorobenzene (TCB) as the eluent (stabilized with $125 \mathrm{ppm}$ BHT). Further details regarding the synthesis of polymers (including GPC and elemental analysis) can be found in the ESI. $\dagger$

UV-vis spectra were measured on glass for the neat polymer films or on ITO/CuSCN for the devices using a Shimadzu UV2600 spectrophotometer.

\subsection{Cyclic voltammetry (CV)}

CV measurements were carried out on solid films using a Bioanalytical Systems (BAS) Epsilon potentiostat with a standard three-electrode configuration. A three electrode cell consisted of a glassy carbon working electrode, $\mathrm{Ag} / \mathrm{AgNO} \mathrm{N}_{3}$ reference electrode and Pt counter electrode. Films were drop-cast onto the glassy carbon electrode from hot chloroform solution $(2 \mathrm{mg}$ $\mathrm{mL}^{-1}$, with tetrabutylammonium hexafluorophosphate added at $100 \mathrm{wt} \%$ relative to polymers) and dried using a heat gun. $0.1 \mathrm{M}$ solution of tetrabutylammonium hexafluorophosphate in anhydrous acetonitrile was used as the supporting electrolyte. Scans were carried out under argon atmosphere at a scan rate of $100 \mathrm{mV} \mathrm{s}^{-1}$. The reference electrode was calibrated using a ferrocene/ferrocenium redox couple. The HOMO (in electron volts) was calculated from the onset of the oxidation potential $\left(E_{\text {ox }}\right)$ according to the following equation:

$$
\mathrm{HOMO}=-\left[4.8 \mathrm{eV}+e\left(E_{\mathrm{Ox}}-E_{\mathrm{Fc} / \mathrm{Fc}^{+}}\right)\right]
$$

\subsection{Photovoltaic device fabrication}

Device fabrication began with cleaning indium tin oxide substrates by sonicating in deionized water, acetone, and isopropyl alcohol followed by UV-ozone treatment for fifteen minutes. CuSCN dissolved in diethylsulfide was spuncast at $7000 \mathrm{rpm}$ to create a $50 \mathrm{~nm}$ film and annealed at $100{ }^{\circ} \mathrm{C}$ for 10 minutes. All subsequent steps were carried out under nitrogen atmosphere. The active layer was spuncast from solutions of polymer(s): $\mathrm{PC}_{61} \mathrm{BM}(1: 2)$ in trichlorobenzene, which were heated for 6 hours at $130{ }^{\circ} \mathrm{C}$. Such conditions are necessary to completely dissolve the monoCNTAZ, which is prone to aggregation and can be difficult to work with at the concentrations necessary for spin casting. $\mathrm{PC}_{61} \mathrm{BM}$ was purchased from Nano-C and used as received. The active layer was then deposited (while still hot) through a $1 \mu \mathrm{m}$ poly(tetrafluoroethylene) filter and spun to achieve the desired film thickness. Films were immediately dried under vacuum for twenty minutes. For the top electrode, $30 \mathrm{~nm}$ of calcium followed by $70 \mathrm{~nm}$ of aluminum were deposited via thermal evaporation at $3 \times 10^{-6}$ mbar. All the photovoltaic devices were measured under AM 1.5G irradi-

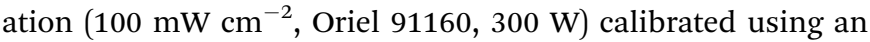
NREL certified standard silicon cell, and recorded using a Keithley 2400 digital source meter. Reported values are the average of at least 6 devices (active area $13 \mathrm{~mm}^{2}$ ) with standard deviation. Thickness of the active layer was measured by an Alpha Step D-100 KLA-Tencor profilometer.

\subsection{Space charge limited current (SCLC)}

To measure the hole mobilities, devices with the structure ITO/ CuSCN/active layer/ $\mathrm{MoO}_{3}(10 \mathrm{~nm}) / \mathrm{Al}(70 \mathrm{~nm})$ were fabricated. The active layer was fabricated under the same conditions used for the photovoltaic devices. Current was measured as a function of voltage in the dark from -1 to $5 \mathrm{~V}$ using a Keithley 2400 digital source meter. Mobilities were calculated according to the Mott-Gurney law:

$$
J=\frac{9}{8} \varepsilon_{\mathrm{r}} \varepsilon_{0} \frac{V^{2}}{d^{3}} \mu
$$

where $\varepsilon_{\mathrm{r}}$ is the dielectric constant of the organic semiconducting material (estimated to be about 3 ), $\varepsilon_{0}$ is the permittivity of free space, and $d$ is the thickness of the film. To calculate the mobility, a fit was applied to the $J^{0.5} v s$. $V$ curve in the SCLC regime.

\subsection{Energy of the CT state}

The CT state was estimated by fitting the equation below to a normalized EQE spectra as described previously. ${ }^{29}$ 


$$
\left.\mathrm{EQE}(E) \propto \frac{E}{\sqrt{4 \pi \lambda k T}} \exp -\frac{\left(E_{\mathrm{CT}}+\lambda-E\right)^{2}}{4 \lambda k T}\right)
$$

\section{Results and discussion}

\subsection{Synthesis}

The terpolymers of these two parent polymers were synthesized at various $\mathrm{mC}: \mathrm{F}$ ratios by controlling the feed ratio of the cyanobenzotriazole monomer, the difluorobenzotriazole monomer, and the benzodithienyl monomer (Scheme 1) as previously reported for the HTAZ:FTAZ system. ${ }^{15,19}$ Incorporation of the cyano and fluorine groups was verified via elemental analysis (see ESI $\dagger$ ). It should be noted that due to the random nature of polymerization, there may be some irregularities along the backbone, including "oligomeric sections" where several BnDTFTAZ units repeat in a row. As such random terpolymers outperformed their regular counterparts in our previous study, the synthetically more difficult regular terpolymer is not investigated here.$^{15}$ For clarity, the terpolymers will be denoted as " $\mathrm{co}$ mC-F 1 : 9" (i.e., copolymer that has a feed ratio of $\mathrm{A} 1$ vs. $\mathrm{A} 2=$ $1: 9$ in Scheme 1), and the ternary blends will simply be "mC:F $1: 9$ " (i.e., blending ratio of monoCTAZ $v s$. FTAZ $=1: 9$, wt $\%$ ).

Cyclic voltammetry was measured for each of the resulting polymers. The terpolymers with greater than 10\% CNTAZ units had similar HOMO levels as that of the monoCNTAZ polymer. These values are tabulated in Table S3 in the ESI. $\dagger$

\subsection{UV-vis absorbance}

We first investigated the effect of blending monoCNTAZ and FTAZ (i.e., terpolymer or ternary blend) on the absorbance of the $\mathrm{BHJ}$ blends based on these polymers (polymer: $\mathrm{PC}_{61} \mathrm{BM}=1: 2$, Fig. 2). In addition, the calculated absorbance coefficients for each ratio (i.e., the weighted average of the FTAZ and monoCNTAZ absorbance) are included for theoretical comparison.

In four of the five ratios (the mC:F $1: 9,3: 7,7: 3$ and $9: 1$ ), the terpolymers and ternary blends show almost identical absorbance peaks and these agree well with the theoretical calculation based on the weighted absorbance of FTAZ and monoCNTAZ. This indicates that the absorbance of the terpolymers and ternary blends is essentially the weighted average of the absorbance of the parent polymers. This would suggest that for most of these blends, there are no significant differences in the absorption (between the terpolymers and ternary blends), which could lead to a change in the photovoltaic behavior. However, the co-mC-F 1:1 terpolymer shows markedly less aggregation from 500-600 $\mathrm{nm}$ than the 1:1 ternary blend. This is a deviation from other ratios, but has been observed in the previously studied DTBT:HTAZ system, where it was suggested that the observed difference in aggregation could lead to low local mobility and performance. ${ }^{15}$ In the ternary blend, the polymers can aggregate separately without interfering with each other; however, in the terpolymer, the random nature of the polymerization increases irregularities along the backbone and decreases aggregation. Nevertheless, given the overall similarities, it is unlikely that absorbance would lead to any difference in the device performance of these systems.

\subsection{Photovoltaic performance}

We further investigated the device performance of all materials in this work. Devices were fabricated in the following conventional structure: ITO/CuSCN/BHJ/Ca/Al. Thickness of the $\mathrm{BHJ}$ layer was maintained at approximately $200 \mathrm{~nm}$ to limit the effects of thickness on the device performance. The performance of all the devices is summarized in Table 1 and the individual device parameters are compared in Fig. 3.

Short circuit current density. The short circuit current density $\left(J_{\mathrm{sc}}\right)$ of the devices based on both ternary blends and terpolymers, where the trend was based on composition, increases as the amount of monoCNTAZ was increased (Fig. 3A). For the mC:F 9:1 and 7:3 ternary blends, the $J_{\mathrm{sc}}$ remained same as that of monoCNTAZ, but as more FTAZ was added (from mC:F $1: 1 \mathrm{on}$ ), the $J_{\text {sc }}$ continued to drop until $1: 9$ ratio, where it was essentially equal to that of FTAZ based devices. This was expected, since monoCNTAZ offers noticeably higher $J_{\text {sc }}$ than FTAZ and its absorption spectrum significantly overlaps with that of FTAZ. Replacing the better light-to-current converter (i.e., monoCNTAZ) with the lesser one (i.e., FTAZ) would thereby lead to a lower $J_{\mathrm{sc}}$ of the ternary blend. Interestingly, when we compare the terpolymers to the ternary blends,

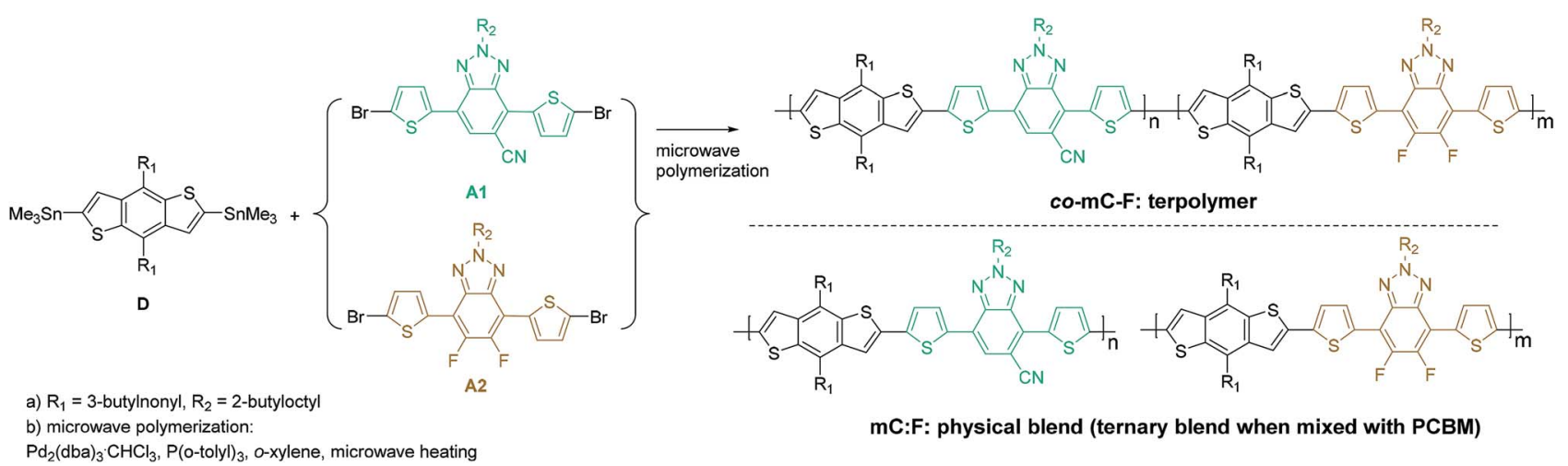

Scheme 1 Synthesis of terpolymers compared with the ternary blend. 

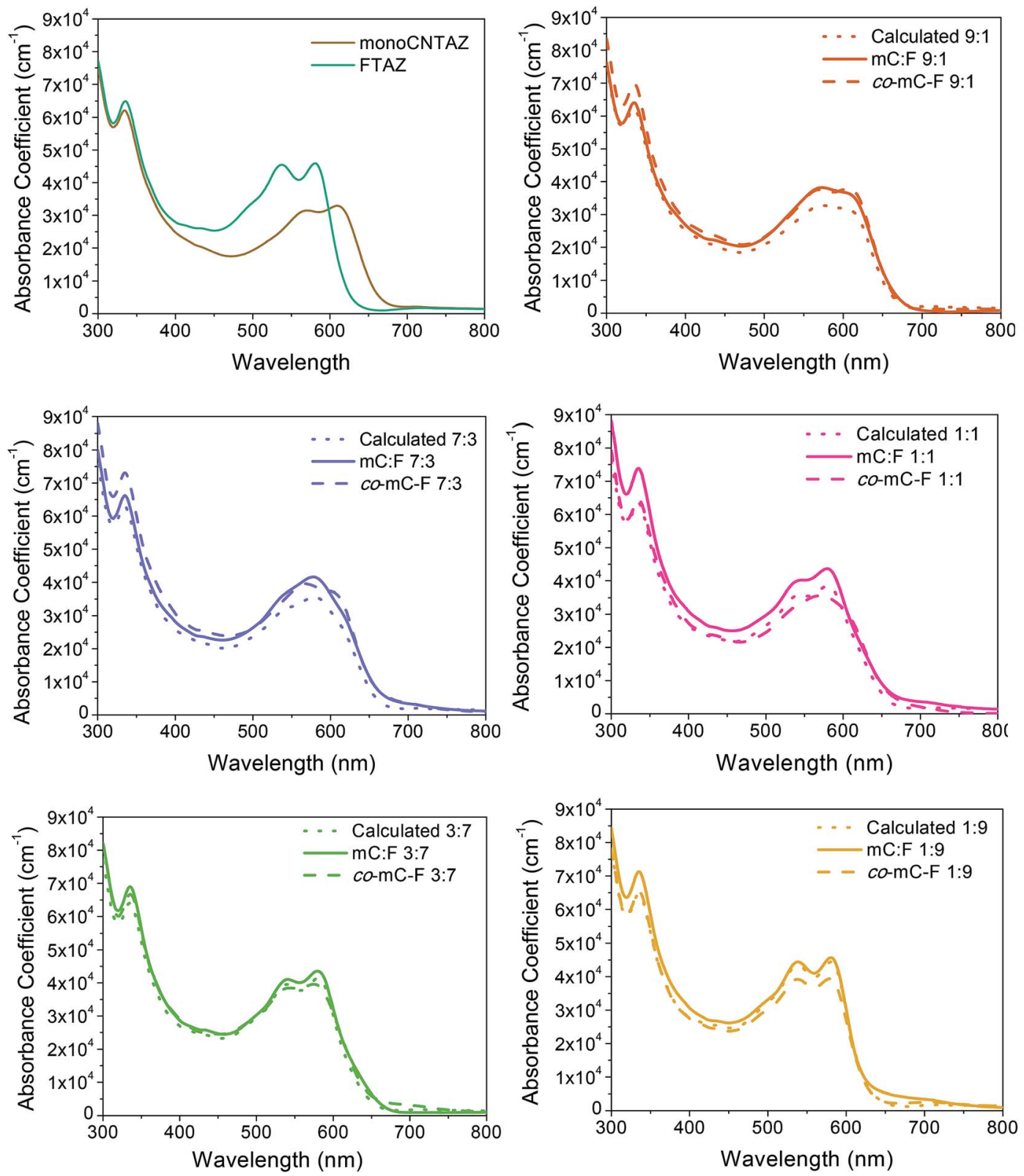

Fig. 2 Absorbance spectra of the $\mathrm{BHJ}$ blends (polymer: $\mathrm{PC}_{61} \mathrm{BM}=1: 2$, weight ratio) for the parent polymers, and all other terpolymers and ternary blends of monoCNTAZ and FTAZ. The solid lines indicate the ternary blends, dashed lines denote the terpolymers, and the dotted lines denote the calculated expected absorbance.

the terpolymers have a lower $J_{\mathrm{sc}}$ across all $\mathrm{mC}: \mathrm{F}$ ratios (Fig. 3A). Given the equivalent absorbance spectra between the terpolymer and ternary blends (except for the $1: 1$ ratio), this difference of $J_{\mathrm{sc}}$ would not stem from a difference in absorbance. Furthermore, the fill factor is maintained across all ratios and not statistically different between terpolymer and ternary blends, which suggests similar charge extraction behavior. ${ }^{16}$ Therefore, another explanation for this significant difference in $J_{\mathrm{sc}}$ is required, and will be posited below. Nevertheless, the difference in $J_{\text {sc }}$ between the terpolymer and the ternary blend at each ratio is not significant $(<10 \%)$.
Open circuit voltage. Both the ternary blends and the terpolymers exhibit composition-dependent $V_{\mathrm{oc}}$, which is a key feature of the parallel-like bulk heterojunction ( $\mathrm{PBHJ}$ ) or the alloy charge generation mechanism (Fig. 3B)..$^{2,30-32}$ This observation indicates that both polymers are participating in charge transport as the $V_{\mathrm{oc}}$ would be pinned if all charges were transported through one polymer. For the ternary blends, the $V_{\mathrm{oc}}$ has a linear dependence on the ratio and agrees with the weighted average of the $V_{o c}$ values of these two binary blends. This offers strong evidence that the ternary blend in this study follows the PBHJ model. ${ }^{4}$ However, for the terpolymers at $7: 3,1: 1$ or $3: 7$ ratios, the value of $V_{\mathrm{oc}}$ is significantly higher than that of the 

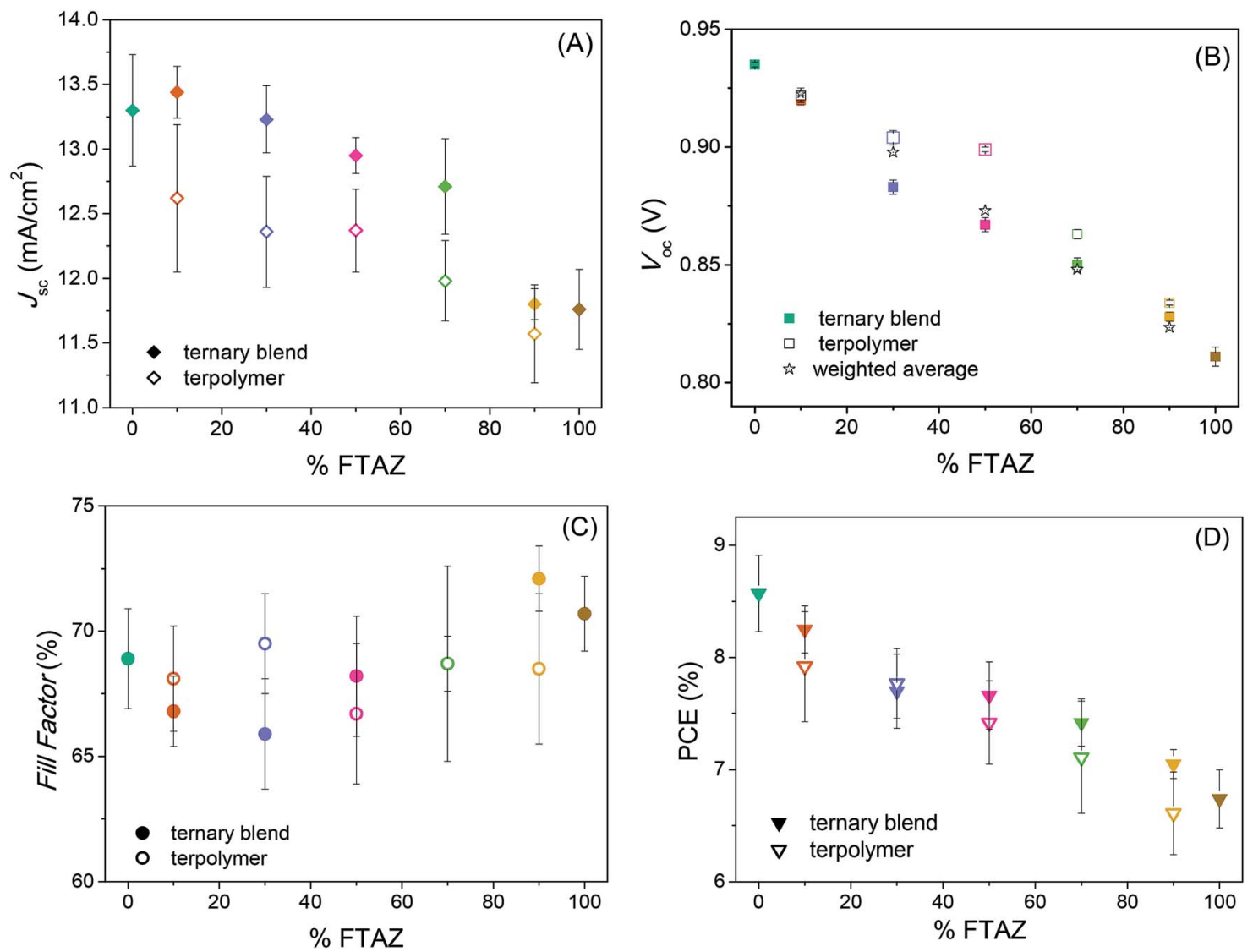

Fig. 3 The photovoltaic device performance of the systems studied: (A) short circuit current, (B) open circuit voltage, (C) fill factor, and (D) power conversion efficiency. Open symbols indicate the terpolymers, while closed symbols denote the ternary blends and the binary parent blends.

corresponding ternary blend. Since our terpolymers mimic the close intermixing of components that would be required for an alloy model, ${ }^{28,30,33}$ the observed difference in $V_{\mathrm{oc}}$ values between the ternary blend and the terpolymer with the identical ratio (i.e., $7: 3$, $1: 1$ or $3: 7)$ implies that the alloy model does not apply in the current system. This is in direct contrast to the previously studied HTAZ:FTAZ system where the values of $V_{\text {oc }}$ of the terpolymer and ternary blend were essentially identical, but has been observed in the previously discussed DTBT:HTAZ system. ${ }^{15}$ This intriguing behavior was further investigated by directly measuring the energy of the charge-transfer state, as discussed below.

Fill factor. Since both polymers have a relatively high FF to start with (above 65\%), the fill factor is maintained in both the terpolymers and the ternary blends (Fig. 3C). While this was somewhat expected, it is to be noted that the difference in backbone between FTAZ and monoCNTAZ could have significantly disrupted the morphology with a corresponding drop in FF. However, there appears no beneficial cooperation in these ternary blends that would lead to higher FF; nevertheless, such increased FF has been seen in other ternary blends. ${ }^{34-37}$

Power conversion efficiency. Since the behavior of both $J_{\mathrm{sc}}$ and $V_{\text {oc }}$ are composition-dependent and FF is maintained, the overall power conversion efficiency (PCE) of both the terpolymers and ternary blends is also composition dependent (Fig. 3D). However, while the efficiency values for the terpolymer and ternary blends are similar, this behavior is not equivalent since it stems from the differences in $V_{\mathrm{oc}}$ behavior and $J_{\mathrm{sc}}$ behavior essentially cancelling each other out. This is different from the HTAZ:FTAZ system, which had equivalent PCE for the terpolymer and ternary blend because of equivalent $V_{\mathrm{oc}}$ and $J_{\mathrm{sc}}$ (and FF).$^{16}$ Whereas, the DTBT:HTAZ system displays different PCE behavior and individual device characteristics $\left(V_{\mathrm{oc}}, J_{\mathrm{sc}}\right.$ and $\mathrm{FF}$ ) for the terpolymer and the ternary blends; $;^{15}$ specifically, the increase in $V_{\text {oc }}$ of the terpolymer could not make up for the decrease in $J_{\mathrm{sc}}$, resulting in an overall lower performance of the terpolymer. These differences in terpolymer $v s$. ternary blend behavior highlight the important nuances of these complex systems. Despite the fact that ultimately both terpolymer and ternary blends achieved the same PCE in this study, the monoCNTAZ:FTAZ system behaves very differently compared to the two systems we studied previously.

External quantum efficiency. While the composition dependent $V_{\mathrm{oc}}$ indicates that both polymers would be contributing to the PCE, the external quantum efficiency (EQE) was measured to confirm this. Fig. 4 includes EQEs of the parent polymers and the $1: 1$ ratio terpolymer and ternary blend (additional EQE are shown in the ESI, Fig. S3†). Notably, both polymers are seen to contribute in the $1: 1$ ratio for both the terpolymer and the ternary blend, with distinct contribution from monoCNTAZ in the $650-700 \mathrm{~nm}$ range and from FTAZ in the $450-500 \mathrm{~nm}$ range. The peak at $700 \mathrm{~nm}$ is from PCBM and has been previously documented. ${ }^{18}$ 


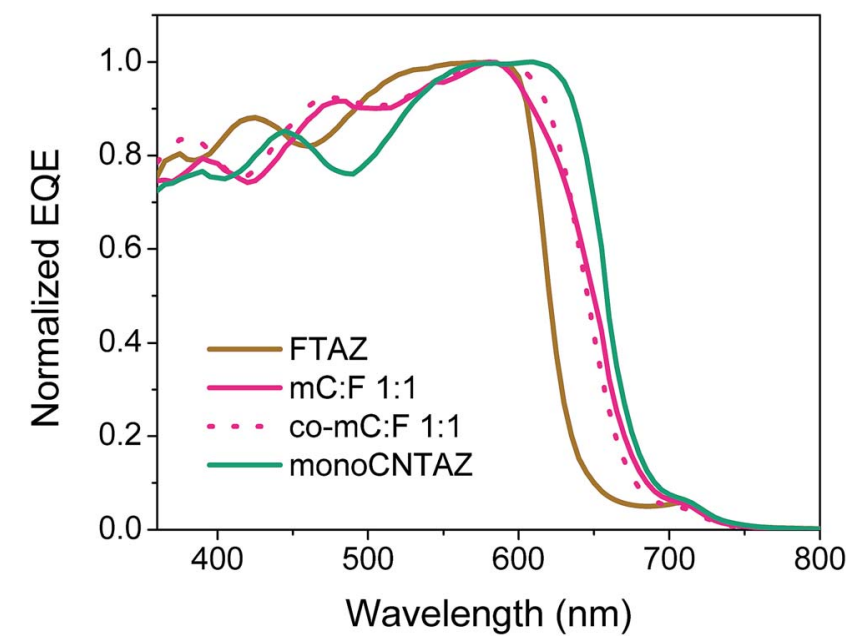

Fig. 4 EQE of the parent binary blends, the $1: 1$ ratio terpolymer and the $1: 1$ ternary blend.

\subsection{Comparison of $V_{\mathrm{oc}}$ and $E_{\mathrm{CT}}$}

In recent years, the importance of the charge transfer (CT) state has come to the forefront in discussions surrounding OSCs, as the energy of the CT state $\left(E_{\mathrm{CT}}\right)$ has a direct effect on the effectiveness of charge transfer within the solar cell and strongly influences the $V_{\text {oc. }}{ }^{\mathbf{3 0 , 3 8 , 3 9}}$ More importantly, it has been a key component in understanding the working mechanism of ternary blends. ${ }^{32}$ Experimentally, we used sensitive, low-energy EQE measurements to directly excite and collect charges from the CT state, as previously described in the literature. ${ }^{\mathbf{3 8 4 0}}$ These EQE were fitted and $E_{\mathrm{CT}}$ was extracted (method described in the ESI $\dagger$ ), which are shown in Fig. 5.

As discussed earlier, the linear dependence of $V_{\text {oc }}$ on the composition (Fig. 3B) indicates that the current ternary blend system probably operates through a $\mathrm{PBHJ}$ mechanism. ${ }^{2,7}$ This PBHJ mechanism has also been supported by other studies. ${ }^{\mathbf{1 1}}$ Further, Savoie et al. applied a density weighted linear combination of the neat polymer density of states (DOS) to estimate the ternary DOS, which could account for the composition dependence of $V_{\mathrm{oc}}$ in the PBHJ cells. ${ }^{4}$ More recently, Felekidis et al. applied a more accurate and sophisticated model to estimate the effective hole DOS at the quasi Fermi level to explain this composition dependence of $V_{\text {oc }}$ in a number of systems. ${ }^{\mathbf{2 1 , 3 1}}$ It appears that the method proposed by Felekidis et al. appears to be the more applicable model at this point, in particular, after the most recent refinement of their model by factoring in the energetic disorder in the donor-acceptor mixed $\mathrm{BHJ}{ }^{21}$

On the other hand, Thompson and the co-workers introduced another model, the organic alloy model, to explain the composition dependency of $V_{\mathrm{oc}}$ in their cases. ${ }^{\mathbf{3 0 , 3 3 , 4 2}}$ They further measured the $E_{\mathrm{CT}}$ of a series of ternary blends and found them to strongly correlate to the observed $V_{\mathrm{oc}} \cdot{ }^{30}$ Subsequently, Kouijzer et al. modelled the $E_{\mathrm{CT}}$ of a series of ternary blends both using an alloy model (where it is assumed an electronic alloy of the two polymers is formed, featuring a delocalized charge transfer state) and a parallel-like bulk heterojunction model (each polymer forms its own charge transfer state and the "averaged" behavior is observed in $\left.V_{\text {oc }}\right) .^{32}$ Though they offered evidence for two distinct CT states in their system, the J$V$ curves of the ternary blends modeled with $\mathrm{PBHJ}$ could only partially explain the observed sub-linear dependence of $V_{\mathrm{oc}}$ on the composition of the ternary blend. When we applied the weighted linear combination of the $E_{\mathrm{CT}}$ to our ternary blends, a clear deviation of the experimental results from the model can be seen in Fig. 5A. This offers evidence that the 'two CT states' model does not truly apply in the current ternary system, either.

Having obtained the $E_{\mathrm{CT}}$ also allows us to investigate more device-related physics. For example, the difference between the lowest optical bandgap in the blend (in this case, $\mathrm{PC}_{61} \mathrm{BM}$ ) and the $E_{\mathrm{CT}}$ of the systems is indicative of the driving force for forming the CT state ( $\left.E_{\mathrm{OPT}}-E_{\mathrm{CT}}\right)$. Interestingly, due to higher $E_{\mathrm{CT}}$ and in turn higher $V_{\mathrm{oc}}$, the terpolymers have a much lower driving force for CT state formation than the ternary blends for each blending ratio (Fig. 5B). This may be one of the contributing factors limiting the $J_{\mathrm{sc}}$ in the case of terpolymers based $\mathrm{BHJ}$ devices, since a lower driving force might lead to slightly lower amounts of CT states formed in the terpolymer. Additionally, by calculating the difference between $E_{\mathrm{CT}}$ and $e V_{\mathrm{oc}}$, we can assess the combined radiative and non-radiative losses in the blends. Fig. 5C shows that there is no definitive trend for the combined losses as a function of ratio; however, for the monoCN:FTAZ $=9: 1,7: 3$, and $1: 1$ ratios, the terpolymers show noticeably more recombination losses than the corresponding ternary blend, which may also contribute to the drop in $J_{\mathrm{sc}}$.
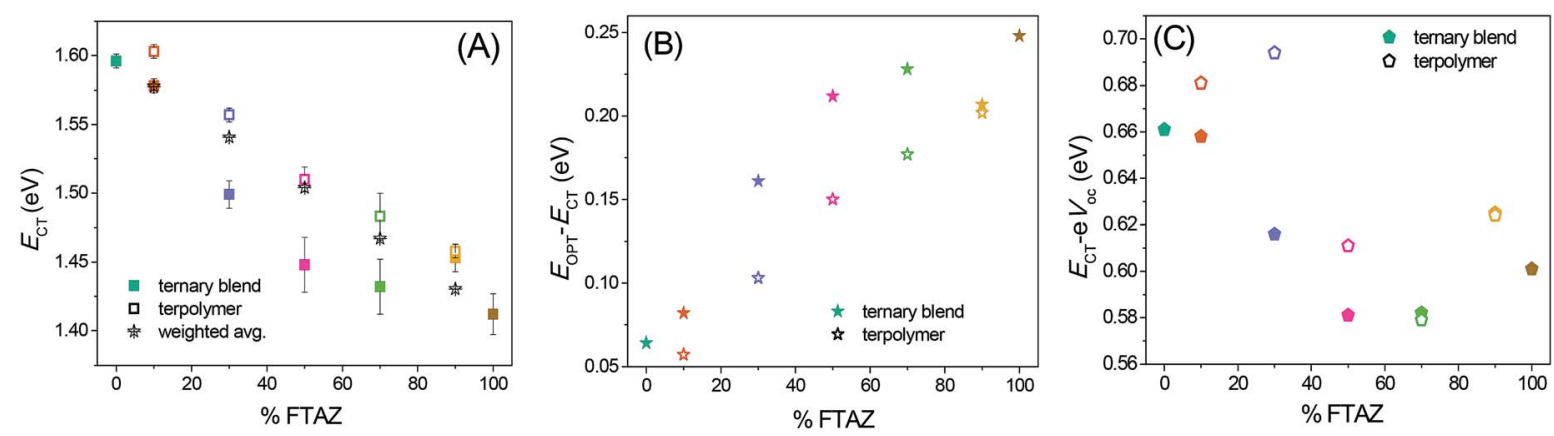

Fig. 5 (A) Energy of the CT state as calculated from low-energy EQE, stars represent the weighted average of CT energies from the parent binary blends, (B) energy difference between the optical bandgap of $\mathrm{PC}_{61} \mathrm{BM}$ (the smallest in the system) and the energy of the $\mathrm{CT}$ state, and (C) energy difference between the energy of the CT state and the observed $V_{\text {oc }}$. 


\subsection{Mobility}

In previous systems featuring FTAZ, physical addition of the fluorinated polymer improved the $\mathrm{FF}$ due to improvement in hole mobility. ${ }^{\mathbf{1 6 , 1 9}}$ Here, since monoCNTAZ already exhibits

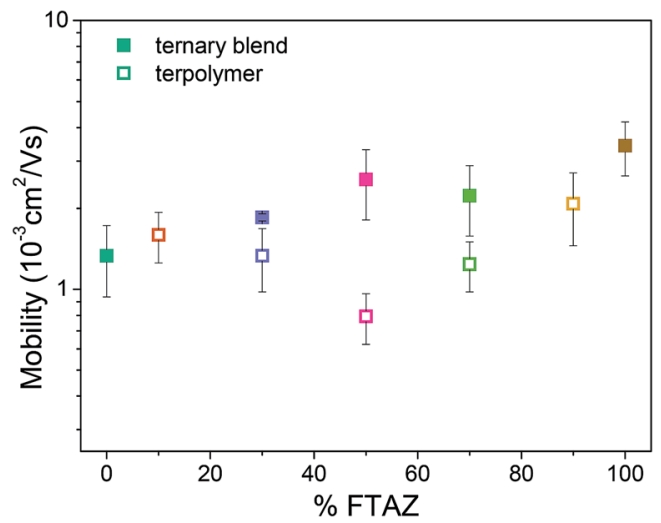

Fig. 6 Hole mobility for selected blends, terpolymers, and binary parent polymers mixed with $\mathrm{PC}_{61} \mathrm{BM}$, and measured via the SCLC method. a mobility similar to FTAZ, we would not expect a significant increase of the hole mobility in the ternary blends. Evidently, as shown in Fig. 6, the hole mobility is maintained at $10^{-3} \mathrm{~cm}^{2} \mathrm{~V}^{-1}$ $\mathrm{s}^{-1}$ for both the terpolymers and the ternary blends. The $1: 1$ terpolymer does show a significantly lower hole mobility than the $1: 1$ ternary blend, but this difference does not seem to have a large effect on the device performance, as their fill factors are not statistically different. This lower hole mobility of the $1: 1$ terpolymer may be due to decrease in aggregation as demonstrated by the UV-vis absorbance spectra.

\subsection{Morphology}

The morphology of the active layer plays an important role in the performance of the solar cell. ${ }^{43}$ Since the performance of a binary solar cell is already dependent on the formation of meta-stable, bi-continuous donor and acceptor domains with a mixed third phase between these two, the addition of a third component further complicates the system. The comparison of the terpolymer blend film morphology to that of the ternary blend is especially interesting for our system, since one might expect the addition of a second polymer (i.e., the tertiary
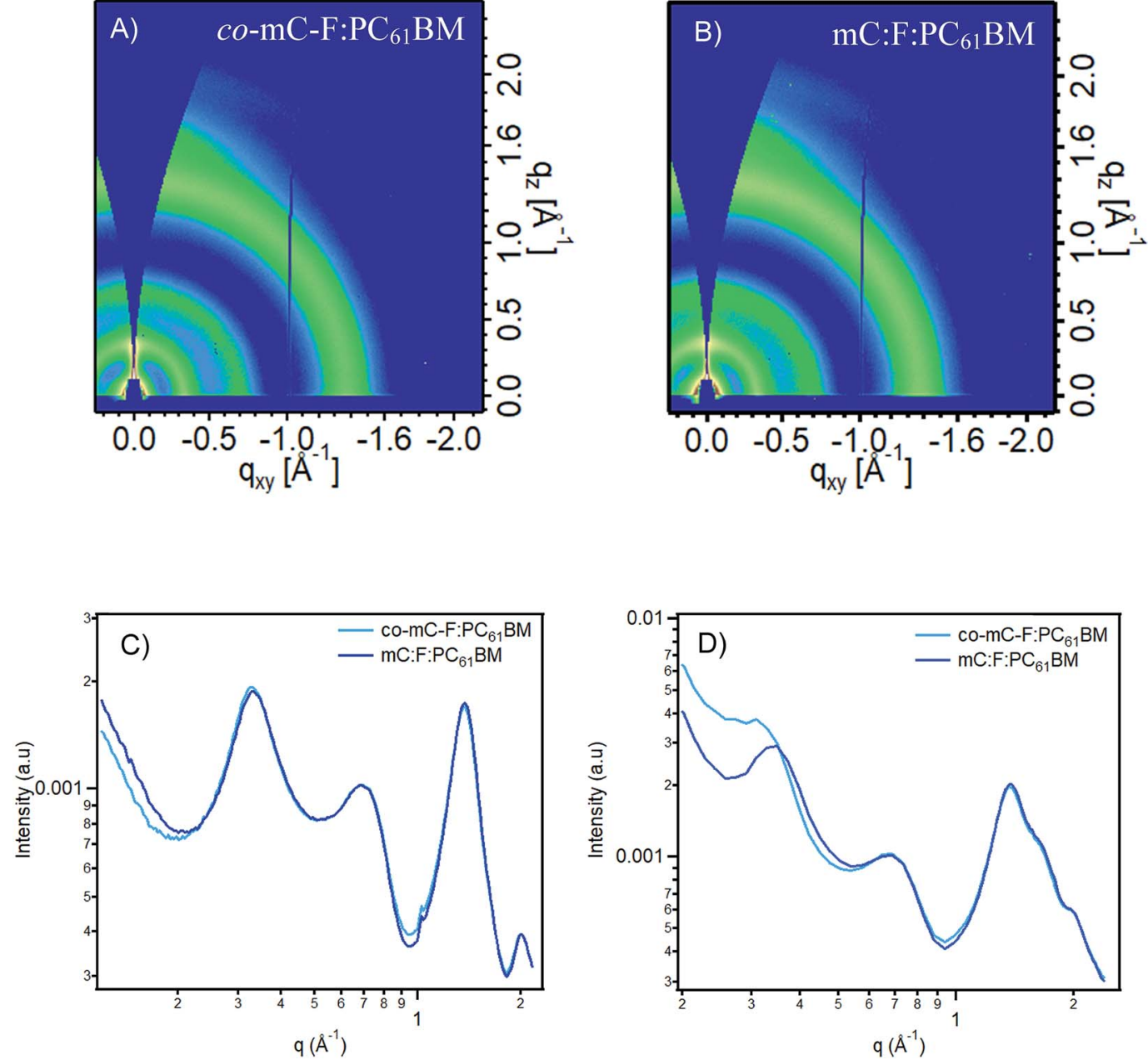

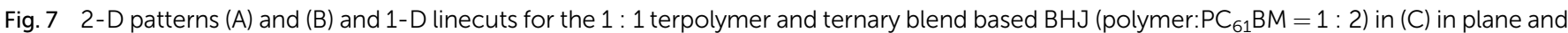
(D) out-of-plane directions. 
Table 2 Peak position and stacking distance as measured by GIWAXS

\begin{tabular}{lll}
\hline BHJ blend & $\begin{array}{l}(010) \text { peak } \\
\text { position }\left(\AA^{-1}\right)\end{array}$ & $\begin{array}{l}\pi-\pi \text { stacking } \\
\text { distance }(\AA)\end{array}$ \\
\hline co-mC-F $1: 1$ & 1.369 & 4.587 \\
mC:F $1: 1$ & 1.370 & 4.584
\end{tabular}

component) to drastically influence domain formation (unless the second polymer was miscible, as has been posited previously). In order to investigate the device morphology, we performed grazing-incidence wide-angle X-ray spectroscopy (GIWAXS), which probes the molecular morphology of the crystalline and semi-crystalline portions of the material, and resonant soft X-ray scattering (RSoXS), which probes the domain spacing and polymer:fullerene composition variations. For simplicity, we have focused on the $1: 1$ terpolymer and $1: 1$ ternary blend (both blended with $\mathrm{PC}_{61} \mathrm{BM}$ ).

GIWAXS. The $2 \mathrm{D}$ patterns of both the terpolymer and the ternary blend are very similar and so are the $1 \mathrm{D}$ linecuts (Fig. 7). This suggests that, despite the slight difference in the aggregation observed in the UV-vis spectra, the molecular packing in these two blends is very similar. Additionally, the pi-pi stacking distance is comparable for both films (Table 2). From these results, the molecular packing probably does not play a role in the in the $J_{\mathrm{sc}}$ or $V_{\mathrm{oc}}$ of these devices.

RSoXS. Measuring the donor: $\mathrm{PC}_{61} \mathrm{BM}$ domains via RSoXS unearthed one of the key differences between the terpolymer and the ternary blend: the domain size. The terpolymer shows a slightly larger domain spacing than the ternary blend, the domain sizes being $82.6 \mathrm{~nm}$ and $62.8 \mathrm{~nm}$, respectively (Fig. 8 and Table 3). This increase in domain size is likely responsible for the lower $J_{\mathrm{sc}}$ of the terpolymer compared to that of the ternary blend (in addition to the lower driving force as revealed via low-energy EQE), as larger domains make it more difficult to effectively harvest the excitons. Additionally, the ternary blend domains have slightly higher purity, which is consistent with the higher FF of the ternary blend (68.2\%) compared to the terpolymer $(66.7 \%)$. Interestingly, despite the addition of a third component, the ternary blend is able to form slightly smaller and purer domains than the terpolymer, thus contributing to

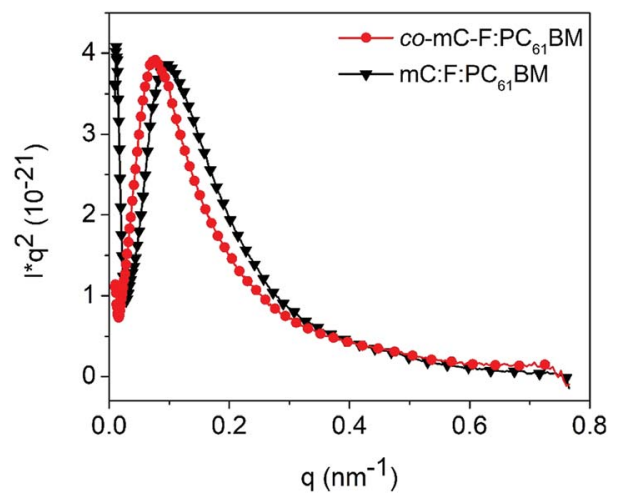

Fig. 8 Lorentz corrected RSoXS (283.2 eV), data normalized for thickness. the $J_{\mathrm{sc}}$. It is worth noting that additional optimization of the processing conditions may decrease the domain size and improve the terpolymer performance.

\section{Conclusion}

This study intended to compare a terpolymer to a ternary blend of two high performing polymers, FTAZ and monoCNTAZ, over a wide range of ratios. It is, in fact, the culmination of a series of studies on such systems. Initially our investigation of the DTBT:HTAZ ternary blend/terpolymer systems (featuring two polymers with structurally different acceptor units in the polymer backbone) led us to believe that terpolymers were unnecessary (in particular, considering the synthetic complexity) since, the ternary blend outperformed the terpolymer in $\mathrm{PC}_{61} \mathrm{BM}$ based $\mathrm{BHJ}$ solar cells in that case. However, additional characterization of the HTAZ:FTAZ system (including a closer look at the charge generation and transport) showed little to no difference between the terpolymer and the ternary blend, a conclusion at odds with our previous assessment. In order to explore this topic further, we present this study, in which two high performance polymers that share a similar backbone (i.e., PBnDT-TAZ) yet contain different substituents (CN and F) are blended into a terpolymer and a ternary blend. These two parent polymers have a significant difference in $V_{\mathrm{oc}}\left(\Delta V_{\mathrm{oc}} \sim 0.13\right.$ $\mathrm{V})$, thereby allowing us to investigate the $V_{\mathrm{oc}}$ changes in the terpolymer $v s$. ternary blend. We measured the $E_{\mathrm{CT}}$ of these ratios and noted that a shift in the $E_{\mathrm{CT}}$ could explain the higher $V_{\text {oc }}$ of the terpolymer, which is a piece of data missing from our previous DTBT:HTAZ study. Therefore, the lower $J_{\mathrm{sc}}$ of the terpolymer based $\mathrm{BHJ}$ blends stems from the lower driving force for CT state formation and the slightly larger and less pure domains. This system serves to highlight the complexity and diversity of such ternary systems and provides a key perspective when viewed in context of our previous studies.

Furthermore, the wide range of ratios studied here makes this system particularly interesting as it considered the differences between the alloy and parallel-like models of charge generation and extraction for ternary blends. The ternary blends in this study show a linear dependence on the blending ratio of the two parent polymers and agree with the weighted average of the $V_{\text {oc }}$ values of the two binary blends. This observation is more in line with the PBHJ model elaborated by Savoie et al. ${ }^{4}$ and the DOS model introduced by Felekidis et al. ${ }^{31}$ Moreover, the trend of measured $E_{\mathrm{CT}}$ with respect to the blending ratio clearly deviates from the weighted linear combination of the $E_{\mathrm{CT}}$ of the two binary blends, which indicates that the "two CT states" model proposed by Kouijzer et al. does not apply to our system.

Table 3 Domain spacing and purity as measured by RSoXS

\begin{tabular}{llll}
\hline Blend & $\begin{array}{l}\text { Peak position } \\
\left(\mathrm{nm}^{-1}\right)\end{array}$ & $\begin{array}{l}\text { Domain spacing } \\
(\mathrm{nm})\end{array}$ & $\begin{array}{l}\text { Average domain } \\
\text { purity }\end{array}$ \\
\hline co-mC-F 1 : 1 & 0.076 & 82.6 & 0.92 \\
mC:F 1 : 1 & 0.100 & 62.8 & 1
\end{tabular}


Thus, the monoCNTAZ:FTAZ ternary blend seems to be governed by a parallel-like mechanism rather than an alloy model.

\section{Conflicts of interest}

There are no conflicts to declare.

\section{Acknowledgements}

M. A. K., Q. Z. and W. Y. were supported by NSF grants (DMR1507249 and CBET-1639429). Work by NCSU was supported by ONR grant N00141512322. X-ray data were acquired at beamlines 11.0.1.2, and 7.3.3 at the Advanced Light Source, which is supported by the Director, Office of Science, Office of Basic Energy Sciences, of the U.S. Department of Energy under Contract No. DE-AC02-05CH11231.

\section{References}

1 H. Huang, L. Yang and B. Sharma, Recent Advances in Organic Ternary Solar Cells, J. Mater. Chem. A, 2017, 5(23), 11501-11517.

2 L. Yang, H. Zhou, S. C. Price and W. You, Parallel-like Bulk Heterojunction Polymer Solar Cells, J. Am. Chem. Soc., 2012, 134(12), 5432-5435.

3 T. Kumari, S. M. Lee, S.-H. Kang, S. Chen and C. Yang, Ternary Solar Cells with a Mixed Face-on and Edge-on Orientation Enable an Unprecedented Efficiency of $12.1 \%$, Energy Environ. Sci., 2017, 10(1), 258-265.

4 B. M. Savoie, S. Dunaisky, T. J. Marks and M. A. Ratner, The Scope and Limitations of Ternary Blend Organic Photovoltaics, Adv. Energy Mater., 2015, 5(3), 1-6.

5 Q. An, F. Zhang, J. Zhang, W. Tang, Z. Deng and B. Hu, Versatile Ternary Organic Solar Cells: A Critical Review, Energy Environ. Sci., 2016, 9(2), 281-322.

6 C. Wang, W. Zhang, X. Meng, J. Bergqvist, X. Liu, Z. Genene, X. Xu, A. Yartsev, O. Inganäs, W. Ma, et al., Ternary Organic Solar Cells with Minimum Voltage Losses, Adv. Energy Mater., 2017, 7(21), 1-10.

7 L. Yang, L. Yan and W. You, Organic Solar Cells beyond One Pair of Donor-Acceptor: Ternary Blends and More, J. Phys. Chem. Lett., 2013, 4(11), 1802-1810.

8 L. Lu, M. A. Kelly, W. You and L. Yu, Status and Prospects for Ternary Organic Photovoltaics, Nat. Photonics, 2015, 9(8), 491-500.

9 H. Li, K. Lu and Z. Wei, Polymer/Small Molecule/Fullerene Based Ternary Solar Cells, Adv. Energy Mater., 2017, 7(17), 1602540.

10 L. Nian, K. Gao, F. Liu, Y. Kan, X. Jiang, L. Liu, Z. Xie, X. Peng, T. P. Russell and Y. Ma, 11\% Efficient Ternary Organic Solar Cells with High Composition Tolerance via Integrated Near-IR Sensitization and Interface Engineering, Adv. Mater., 2016, 28(37), 8184-8190.

11 Z. Xiao, X. Jia and L. Ding, Ternary Organic Solar Cells Offer 14\% Power Conversion Efficiency, Sci. Bull., 2017, 62(23), 1562-1564.
12 N. S. Gobalasingham, S. Noh, J. B. Howard and B. C. Thompson, Influence of Surface Energy on Organic Alloy Formation in Ternary Blend Solar Cells Based on Two Donor Polymers, ACS Appl. Mater. Interfaces, 2016, 8(41), 27931-27941.

13 L. Xiao, T. Liang, K. Gao, T. Lai, X. Chen, F. Liu, T. P. Russell, F. Huang, X. Peng and Y. Cao, Ternary Solar Cells Based on Two Small Molecule Donors with Same Conjugated Backbone: The Role of Good Miscibility and Hole Relay Process, ACS Appl. Mater. Interfaces, 2017, 9(35), 2991729923.

14 Y. Yang, W. Chen, L. Dou, W.-H. Chang, H.-S. Duan, B. Bob, G. Li and Y. Yang, High-Performance Multiple-Donor Bulk Heterojunction Solar Cells, Nat. Photonics, 2015, 9(3), 190198.

15 Q. Zhang, M. A. Kelly, A. Hunt, H. Ade and W. You, Comparative Photovoltaic Study of Physical Blending of Two Donor-Acceptor Polymers with the Chemical Blending of the Respective Moieties, Macromolecules, 2016, 49(7), 2533-2540.

16 M. A. Kelly, S. Roland, Q. Zhang, Y. Lee, B. Kabius, Q. Wang, E. D. Gomez, D. Neher and W. You, Incorporating Fluorine Substitution into Conjugated Polymers for Solar Cells: Three Different Means, Same Results, J. Phys. Chem. C, 2017, 121(4), 2059-2068.

17 J. R. Tumbleston, A. C. Stuart, E. Gann, W. You and H. Ade, Fluorinated Polymer Yields High Organic Solar Cell Performance for a Wide Range of Morphologies, Adv. Funct. Mater., 2013, 23(27), 3463-3470.

18 S. C. Price, A. C. Stuart, L. Yang, H. Zhou and W. You, Fluorine Substituted Conjugated Polymer of Medium Band Gap Yields 7\% Efficiency in Polymer-Fullerene Solar Cells, J. Am. Chem. Soc., 2011, 133(12), 4625-4631.

19 W. Li, S. Albrecht, L. Yang, S. Roland, J. R. Tumbleston, T. McAfee, L. Yan, M. A. Kelly, H. Ade, D. Neher, et al., Mobility-Controlled Performance of Thick Solar Cells Based on Fluorinated Copolymers, J. Am. Chem. Soc., 2014, 136(44), 15566-15576.

20 N. D. Eastham, A. S. Dudnik, B. Harutyunyan, T. J. Aldrich, M. J. Leonardi, E. F. Manley, M. R. Butler, T. Harschneck, M. A. Ratner, L. X. Chen, et al., Enhanced Light Absorption in Fluorinated Ternary Small-Molecule Photovoltaics, ACS Energy Lett., 2017, 2(7), 1690-1697.

21 N. Felekidis, A. Melianas and M. Kemerink, Design Rule for Improved Open-Circuit Voltage in Binary and Ternary Organic Solar Cells, ACS Appl. Mater. Interfaces, 2017, 9(42), 37070-37077.

22 W. Li, L. Yan, H. Zhou and W. You, A General Approach toward Electron Deficient Triazole Units to Construct Conjugated Polymers for Solar Cells, Chem. Mater., 2015, 27(18), 6470-6476.

23 T. Ameri, P. Khoram, J. Min and C. J. Brabec, Organic Ternary Solar Cells: A Review, Adv. Mater., 2013, 21, 42454266.

24 Y. Gu, C. Wang, F. Liu, J. Chen, O. E. Dyck, G. Duscher, T. P. Russell, L. Yu, M. Yuan, M. Baklar, et al., Guided Crystallization of P3HT in Ternary Blend Solar Cell Based 
on P3HT:PCPDTBT:PCBM, Energy Environ. Sci., 2014, 7(11), 3782-3790.

25 L. Lu, T. Xu, W. Chen, E. S. Landry and L. Yu, Ternary Blend Polymer Solar Cells with Enhanced Power Conversion Efficiency, Nat. Photonics, 2014, 8(9), 716-722.

26 S. Liu, P. You, J. Li, J. Li, C.-S. Lee, B. S. Ong, C. Surya and F. Yan, Enhanced Efficiency of Polymer Solar Cells by Adding a High-Mobility Conjugated Polymer, Energy Environ. Sci., 2015, 8(5), 1463-1470.

27 B. Xia, L. Yuan, J. Zhang, Z. Wang, J. Fang, Y. Zhao, D. Deng, W. Ma, K. Lu and Z. Wei, Evolution of Morphology and Open-Circuit Voltage in Alloy-Energy Transfer Coexisting Ternary Organic Solar Cells, J. Mater. Chem. A, 2017, 5(20), 9859-9866.

28 P. P. Khlyabich, A. E. Rudenko, B. C. Thompson and Y.-L. Loo, Structural Origins for Tunable Open-Circuit Voltage in Ternary-Blend Organic Solar Cells, Adv. Funct. Mater., 2015, 25(34), 5557-5563.

29 E. T. Hoke, K. Vandewal, J. A. Bartelt, W. R. Mateker, J. D. Douglas, R. Noriega, K. R. Graham, J. M. J. Fréchet, A. Salleo and M. D. McGehee, Recombination in Polymer:Fullerene Solar Cells with Open-Circuit Voltages Approaching and Exceeding $1.0 \mathrm{~V}$, Adv. Energy Mater., 2013, 3(2), 220-230.

30 R. A. Street, D. Davies, P. P. Khlyabich, B. Burkhart and B. C. Thompson, Origin of the Tunable Open-Circuit Voltage in Ternary Blend Bulk Heterojunction Organic Solar Cells, J. Am. Chem. Soc., 2013, 135(3), 986-989.

31 N. Felekidis, E. Wang and M. Kemerink, Open Circuit Voltage and Efficiency in Ternary Organic Photovoltaic Blends, Energy Environ. Sci., 2016, 9(1), 257-266.

32 S. Kouijzer, W. Li, M. M. Wienk and R. A. J. Janssen, Charge Transfer State Energy in Ternary Bulk-Heterojunction Polymer-fullerene Solar Cells, J. Photonics Energy, 2014, 5(1), 057203.

33 P. P. Khlyabich, M. Sezen-Edmonds, J. B. Howard, B. C. Thompson and Y. L. Loo, Formation of Organic Alloys in Ternary-Blend Solar Cells with Two Acceptors Having Energy-Level Offsets Exceeding 0.4 eV, ACS Energy Lett., 2017, 2(9), 2149-2156.

34 J. Lee, V. Tamilavan, K. H. Rho, S. Keum, K. H. Park, D. Han, Y. K. Jung, C. Yang, Y. Jin, J. W. Jang, et al., Overcoming Fill Factor Reduction in Ternary Polymer Solar Cells by Matching the Highest Occupied Molecular Orbital Energy
Levels of Donor Polymers, Adv. Energy Mater., 2018, 8(9), 1702251.

35 W. Li, Y. Yan, Y. Gong, J. Cai, F. Cai, R. S. Gurney, D. Liu, A. J. Pearson, D. G. Lidzey and T. Wang, Contrasting Effects of Energy Transfer in Determining Efficiency Improvements in Ternary Polymer Solar Cells, Adv. Funct. Mater., 2017, 28(5), 1704212.

36 N. Gasparini, X. Jiao, T. Heumueller, D. Baran, G. J. Matt, S. Fladischer, E. Spiecker, H. Ade, C. J. Brabec and T. Ameri, Designing Ternary Blend Bulk Heterojunction Solar Cells with Reduced Carrier Recombination and a Fill Factor of 77\%, Nat. Energy, 2016, 1(9), 1-9.

37 N. Gasparini, L. Lucera, M. Salvador, M. Prosa, G. D. Spyropoulos, P. Kubis, H.-J. Egelhaaf, C. J. Brabec and T. Ameri, High-Performance Ternary Organic Solar Cells with Thick Active Layer Exceeding 11\% Efficiency, Energy Environ. Sci., 2017, 10(4), 885-892.

38 K. Vandewal, K. Tvingstedt, A. Gadisa, O. Inganäs and J. V. Manca, Relating the Open-Circuit Voltage to Interface Molecular Properties of Donor:Acceptor Bulk Heterojunction Solar Cells, Phys. Rev. B: Condens. Matter Mater. Phys., 2010, 81(12), 125204.

39 T. M. Clarke and J. R. Durrant, Charge Photogeneration in Organic Solar Cells, Chem. Rev., 2010, 110(11), 6736-6767.

40 N. Bauer, Q. Zhang, J. Zhao, L. Ye, J.-H. Kim, I. Constantinou, L. Yan, F. So, H. Ade, H. Yan, et al., Comparing NonFullerene Acceptors with Fullerene in Polymer Solar Cells: A Case Study with FTAZ and PyCNTAZ, J. Mater. Chem. A, 2017, 5(10), 4886-4893.

41 T. H. Lee, M. A. Uddin, C. Zhong, S.-J. Ko, B. Walker, T. Kim, Y. J. Yoon, S. Y. Park, A. J. Heeger, H. Y. Woo, et al., Investigation of Charge Carrier Behavior in High Performance Ternary Blend Polymer Solar Cells, Adv. Energy Mater., 2016, 6(19), 1600637.

42 P. P. Khlyabich, A. E. Rudenko, R. A. Street and B. C. Thompson, Influence of Polymer Compatibility on the Open-Circuit Voltage in Ternary Blend Bulk Heterojunction Solar Cells, ACS Appl. Mater. Interfaces, 2014, 6(13), 9913-9919.

43 X. Jiao, L. Ye and H. Ade, Quantitative MorphologyPerformance Correlations in Organic Solar Cells: Insights from Soft X-Ray Scattering, Adv. Energy Mater., 2017, 7(18), 1700084. 